\title{
A simple method for tuning the optical properties of thermoresponsive plasmonic nanogels
}

Fei Han, ${ }^{1}$ Alexander H. Soeriyadi, ${ }^{1,2}$ S. R. C. Vivekchand, ${ }^{1}$ J. Justin Gooding ${ }^{1,2,3, *}$

${ }^{1}$ School of Chemistry, The University of New South Wales, Sydney, New South Wales 2052, Australia

${ }^{2}$ Australian Centre for Nanomedicine, The University of New South Wales, Sydney, New South Wales 2052, Australia

${ }^{3} \mathrm{ARC}$ Center of Excellence in Convergent Bio-Nano Science and Technology, The University of New South Wales, Sydney, New South Wales 2052, Australia

"corresponding author: justin.gooding@unsw.edu.au 


\section{Experimental Methods}

\section{Materials}

The $\mathrm{Au}$ nanoparticles (with average diameters of $20 \mathrm{~nm}, 40 \mathrm{~nm}, 80 \mathrm{~nm}$ ) were purchased from BBI solutions (Cardiff, United Kingdom). 2(dodecylthiocarbonothioylthio)-2-methylpropionic acid (DDMAT) (HPLC, 98\%), Tween $20(0.06 \mathrm{mM})$, sodium chloride (ACS, $\geq 99.0 \%)$, and chloroform-D $\left(\mathrm{CDCl}_{3}\right)$ (99.8 atom \%D) were purchased from Sigma-Aldrich (Sydney, Australia) and used as received. 1,4-Dioxane (HPLC, 98\%) was bought from the Honeywell, Burdick \& Jackson HPLC certified solvent (Adelaide, Australia). Monomer $\mathrm{N}$ isopropylacrylamide (NIPAM) (97\%) was recrystallized from ethanol before used. 2,2'-azobis(2-methylpropionitrile) (AIBN) (98\%) was recrystallized from methanol before used.

\section{RAFT polymerization of NIPAM}

In a typical polymerization, NIPAM (2.50 g, $22.1 \mathrm{mmol})$, DDMAT (40.3 mg, 0.110 $\mathrm{mmol})$, AIBN and 1,4-dioxane $(8 \mathrm{~mL})$ were sealed in a $250 \mathrm{~mL}$ vial equipped with a magnetic stirrer bar and rubber septum, and then charged into a Schlenk flask. The solution was purged with nitrogen for $30 \mathrm{~min}$ and followed by stirring under nitrogen in a pre-heated oil bath at $65{ }^{\circ} \mathrm{C}$. The samples were removed periodically by syringe to determine the molecular weight and monomer conversion by ${ }^{1} \mathrm{H}$ NMR spectroscopy. The reaction mixture was exposed to air and quenched in an ice bath. The solution was diluted with a small amount of THF and precipitated into hexane. The resulting yellow precipitate was collected by centrifugation, redissolved in $2 \mathrm{~mL}$ of THF and precipitated into hexane again. This purification process was repeated five times in total. The purified polymer was collected and dried under vacuum to yield pNIPAM as a yellow solid.

\section{Functonalization of AuNPs with pNIPAM}

In a typical procedure, AuNP (80 nm diameter, $500 \mu \mathrm{L})$ solution was concentrated to $10 \mu \mathrm{L}$ by centrifugation at $3500 \mathrm{rpm}$ for $20 \mathrm{~min}$. In the next step, pNIPAM solution (dissolved in water) $(10 \mu \mathrm{L}, 1.8 \mathrm{mM})$ was added into the AuNP solution ([pNIPAM] : $[$ AuNP $\left.]=10^{7}: 1\right)$. To this mixture, Tween $20(2 \mu \mathrm{L}, 2.5 \%)$ and Milli-Q water $(28 \mu \mathrm{L})$ was added and left to react. After $1 \mathrm{~h}$, sodium chloride solution $(4 \mu \mathrm{L}, 0.2 \mathrm{M})$ was added to this mixture and the solution was left to stand without stirring for a further 1 h. Finally, the total volume was increased to $100 \mu \mathrm{L}$ by the repeated addition of $\mathrm{NaCl}$ solution $(4 \mu \mathrm{L}, 0.2 \mathrm{M})$ in every half hour and the solution was incubated at $600 \mathrm{rpm}$ 
overnight. The excess polymer and $\mathrm{NaCl}$ was removed by centrifugation $(3500 \mathrm{rpm}$ in $20 \mathrm{~min}$, repeated twice). The final resultant was diluted to the $500 \mu \mathrm{L}$.

\section{Preparation of the responsive plasmonic nanohybrids}

In a typical procedure, the certain amount of the pNIPAM matrix solutions $\left(M_{\mathrm{w}}=\right.$ 46,500, $1.8 \mathrm{mM}$ ) was added into the $100 \mu \mathrm{L}$ pNIPAM@AuNP solution to form the plasmonic nanohybrids in Milli-Q water. The mixture was gently agitated for $15 \mathrm{~min}$. The corresponding content of each nanohybrid sample is shown in Table 1.

\section{Characterization}

The NMR spectra of pNIPAM prepared by RAFT polymerization was recorded on a Bruker Avance III $300 \mathrm{MHz}$ spectrometer with deuterated chloroform $\left(\mathrm{CDCl}_{3}\right)$ as the solvent. All gel permeation chromatography (GPC) measurements in this project were performed Shimadzu modular system (LC20-AT plunger, SIL-10AD autoinjector, CTO-16AC column oven and RID-10A RI detector) at a constant temperature of $40{ }^{\circ} \mathrm{C}$. HPLC grade $N, N$-dimethyl acetamide (DMAc) is used as the

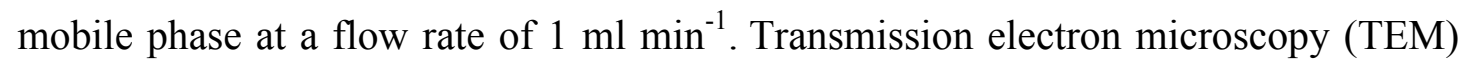
images of the AuNPs and the nanohybrids were obtained with the FEI TECNAI G2 transmission electron microscope at an acceleration voltage of $200 \mathrm{kV}$. A small drop of AuNP solution was first deposited on a Formvar/carbon-coated copper grid, and dried at the room temperature. The TEM images of AuNPs are shown in the Fig. S1. For the nanohybrids samples, the TEM grid was prepared at $40{ }^{\circ} \mathrm{C}$. In this procedure, the pipette, pipette tip, copper grid as well as the pNIPAM-AuNP nanohybrid solution was preheated at $40{ }^{\circ} \mathrm{C}$ for $10 \mathrm{~min}$. Subsequently, a small drop of the pNIPAM-AuNP nanohybrid solution was deposited onto the $\mathrm{Cu}$ grid, and the solution was left to dry at $40{ }^{\circ} \mathrm{C}$.

The UV-visible absorption spectra were collected with a Lambda Cary 1050 spectrophotometer. A cuvette with $2 \mathrm{~mm}$ path length was employed and the spectra were recorded in the $400-850 \mathrm{~nm}$ range. Temperature of the sample was controlled via an integrated Peltier heater, the samples were heated with a heating rate of $2^{\circ} \mathrm{C} / \mathrm{min}$. Dynamic light scattering (DLS) measurements were performed on a Malvern Zetasizer Nano Series with a laser light of wavelength $633 \mathrm{~nm}$. The finite difference time-domain (FDTD) calculation in the study were performed with Lumerical Solutions FDTD simulations. 
Table S1. Summary of the corresponding content of the Nanohybrids and pNIPAM@AuNP.

\begin{tabular}{|c|c|}
\hline Sample & Content \\
\hline Nanogel 1 & pNIPAM@AuNP 20 \\
\hline Nanogel 2 & pNIPAM@AuNP 40 \\
\hline Nanogel 3 & pNIPAM@AuNP 80 \\
\hline Nanogel 4 & pNIPAM@AuNP ${ }_{20}$ in the pNIPAM matrix solution \\
\hline Nanogel 5 & pNIPAM@AuNP 40 in the pNIPAM matrix solution \\
\hline Nanogel 6 & pNIPAM@AuNP s0 $_{80}$ in the pNIPAM matrix solution \\
\hline Nanogel 7 & pNIPAM@AuNP ${ }_{20}+$ pNIPAM@AuNP $\mathbf{P}_{80}$ in the pNIPAM matrix solution \\
\hline Nanogel 8 & pNIPAM@AuNP $40+$ pNIPAM@AuNP An $_{80}$ in the pNIPAM matrix solution \\
\hline Nanogel 9 & pNIPAM@AuNP ${ }_{20}+$ pNIPAM@AuNP 4 in the pNIPAM matrix solution \\
\hline
\end{tabular}


(a)

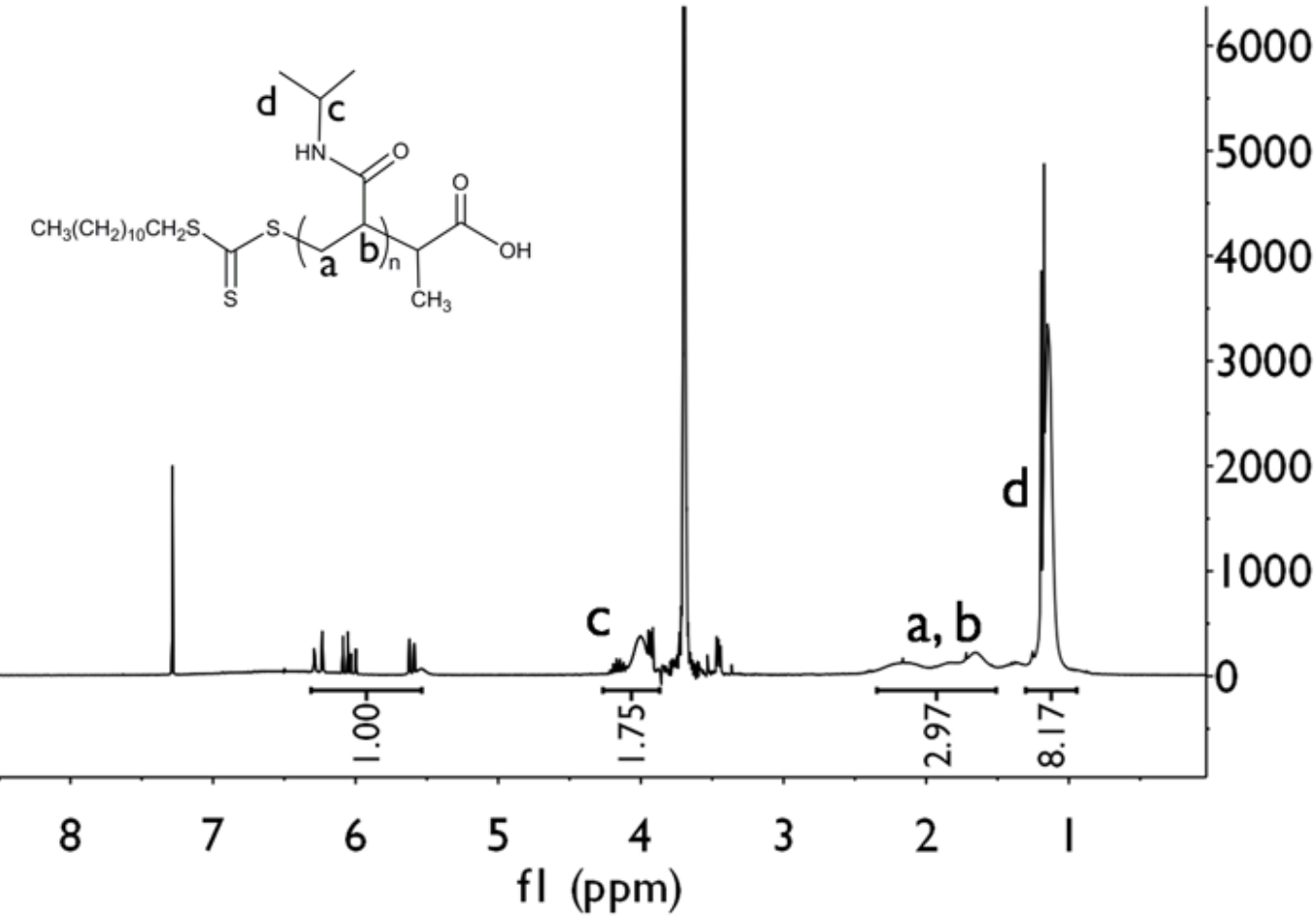

(b)

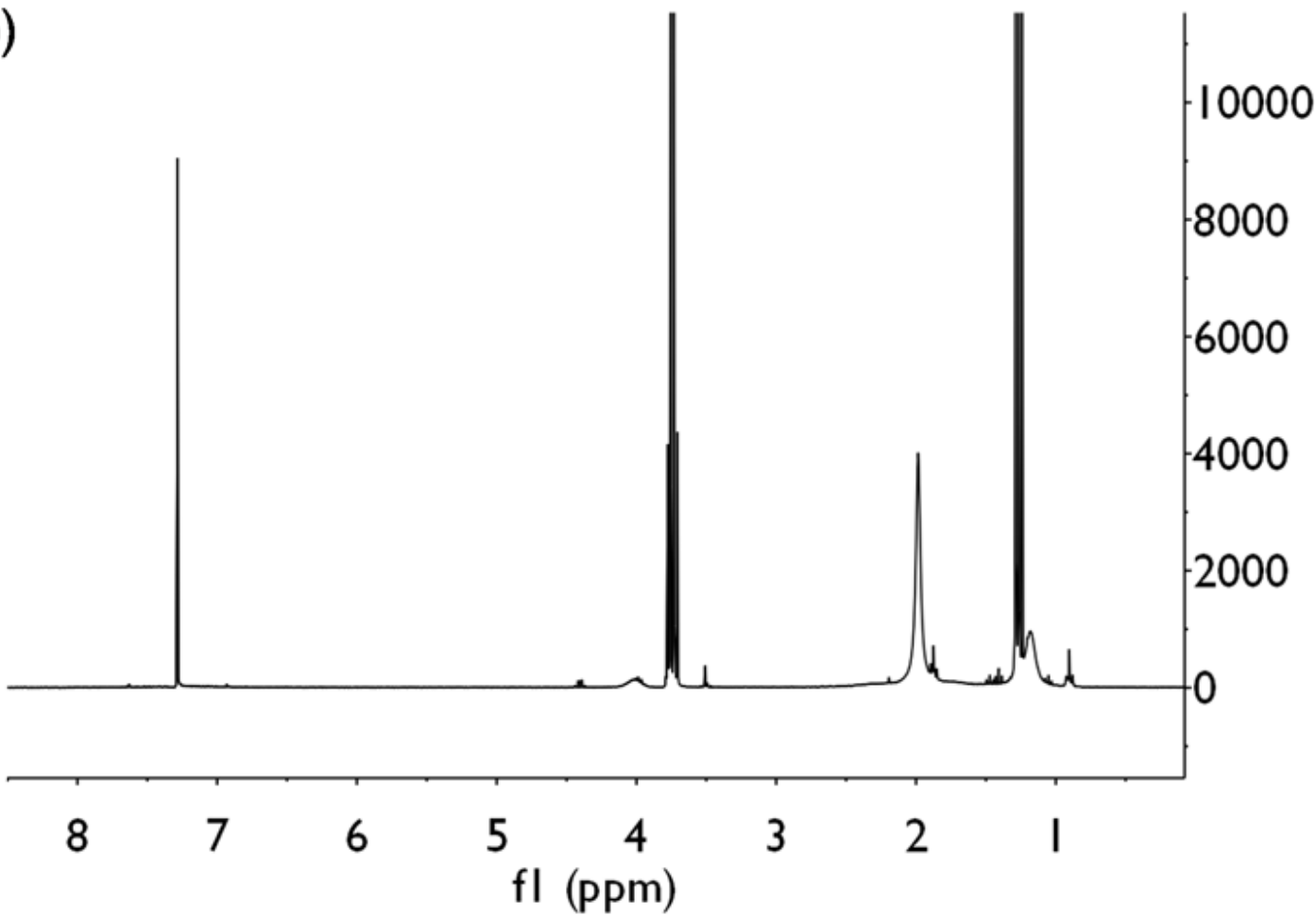

Figure S1. ${ }^{1} \mathrm{H}$ NMR spectra and peak labels for pNIPAM: (a) before purification and (b) after purification. Characteristic signals of pNIPAM at c) 3.98 and d) $1.21 \mathrm{ppm}$ representing methyne and methyl protons on isopropyl groups were observed, which verified the successful synthesis of pNIPAM chains. 


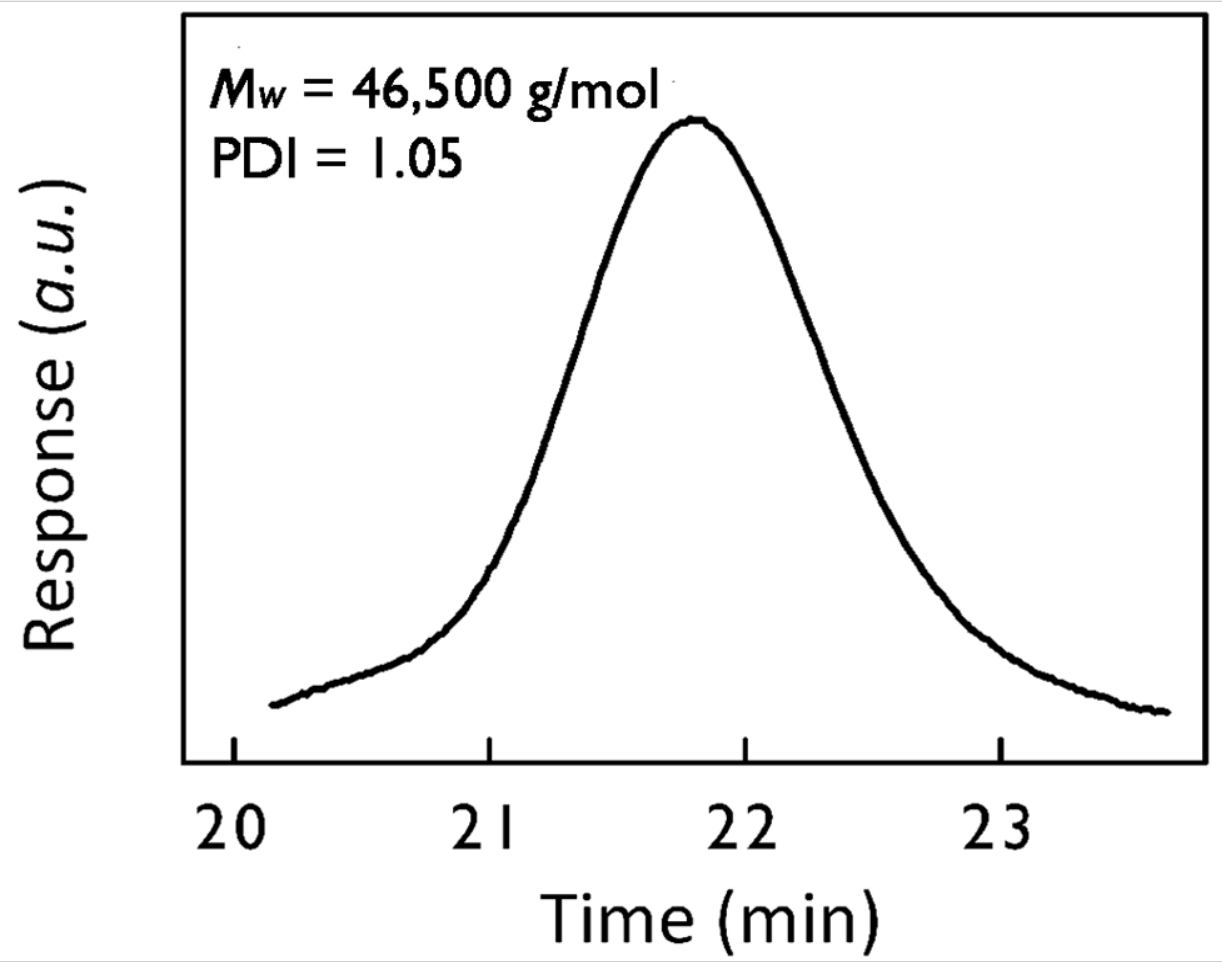

Figure S2. GPC traces of pNIPAM $\left(M_{\mathrm{w}}=46,500 \mathrm{~g} / \mathrm{mol}, \emptyset=1.05\right)$.

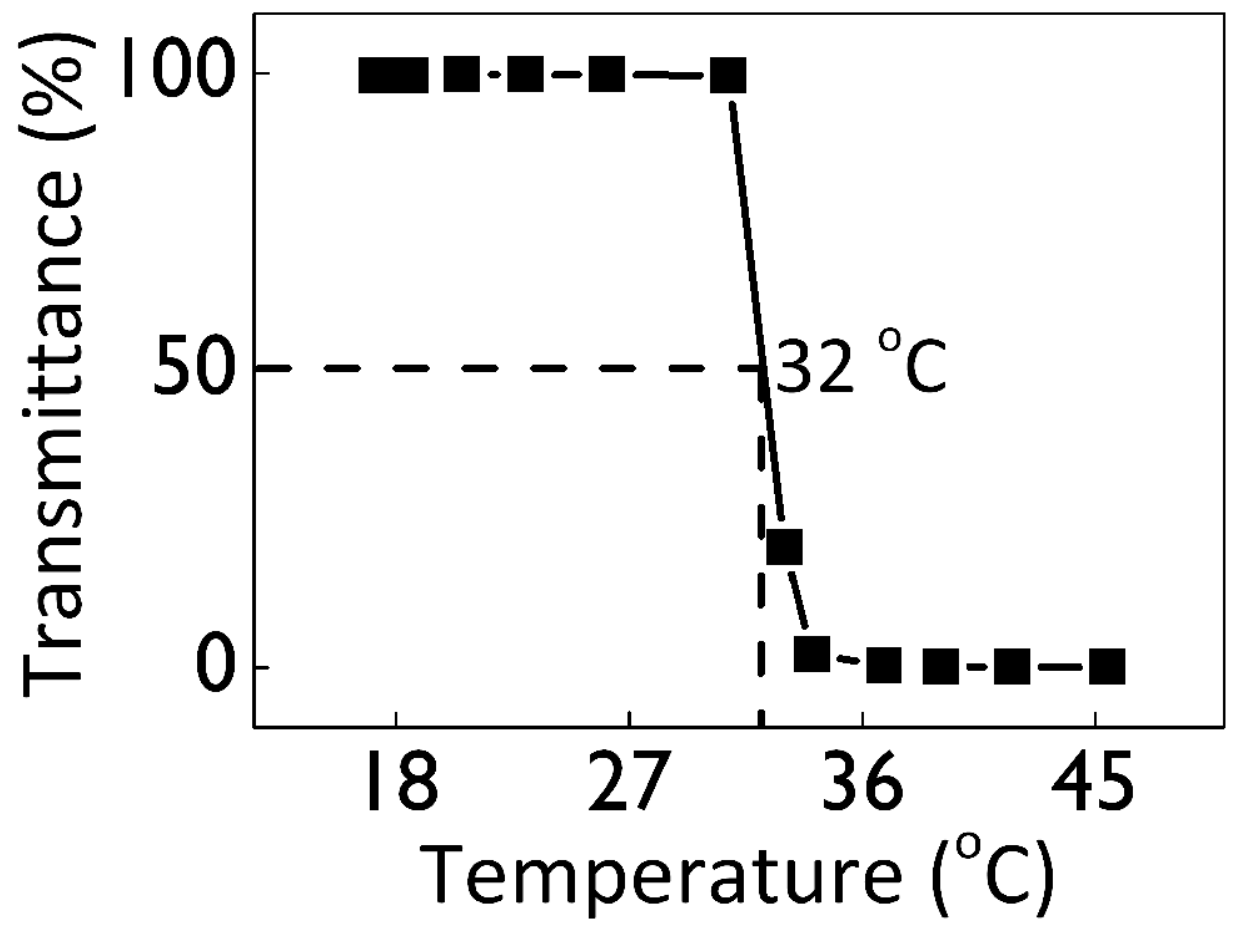

Figure S3. Thermal Profile $\left(2{ }^{\circ} \mathrm{C} / \mathrm{min}\right)$ of the RAFT synthesized pNIPAM $\left(M_{\mathrm{w}}=46,500\right)$ in MilliQ water as measured by UV-Vis $([\mathrm{pNIPAM}]=5 \mathrm{mg} / \mathrm{mL})$. The lower critical solution temperature of pNIPAM is $32{ }^{\circ} \mathrm{C}$. 

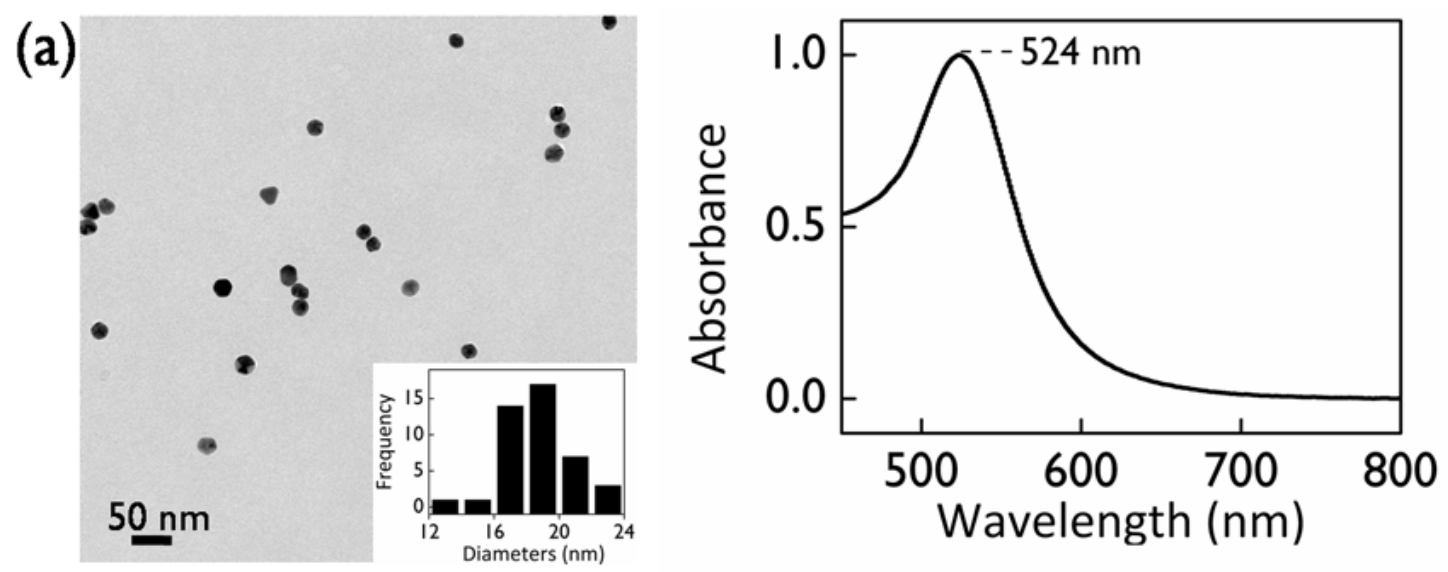

(b)
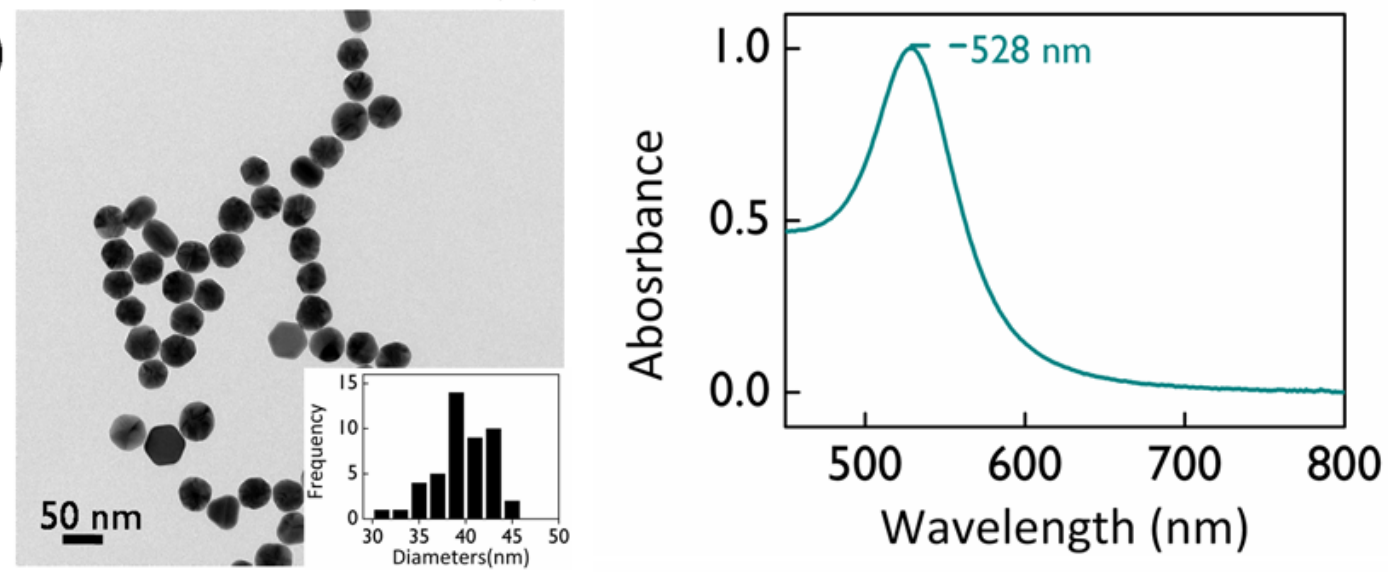

(c)
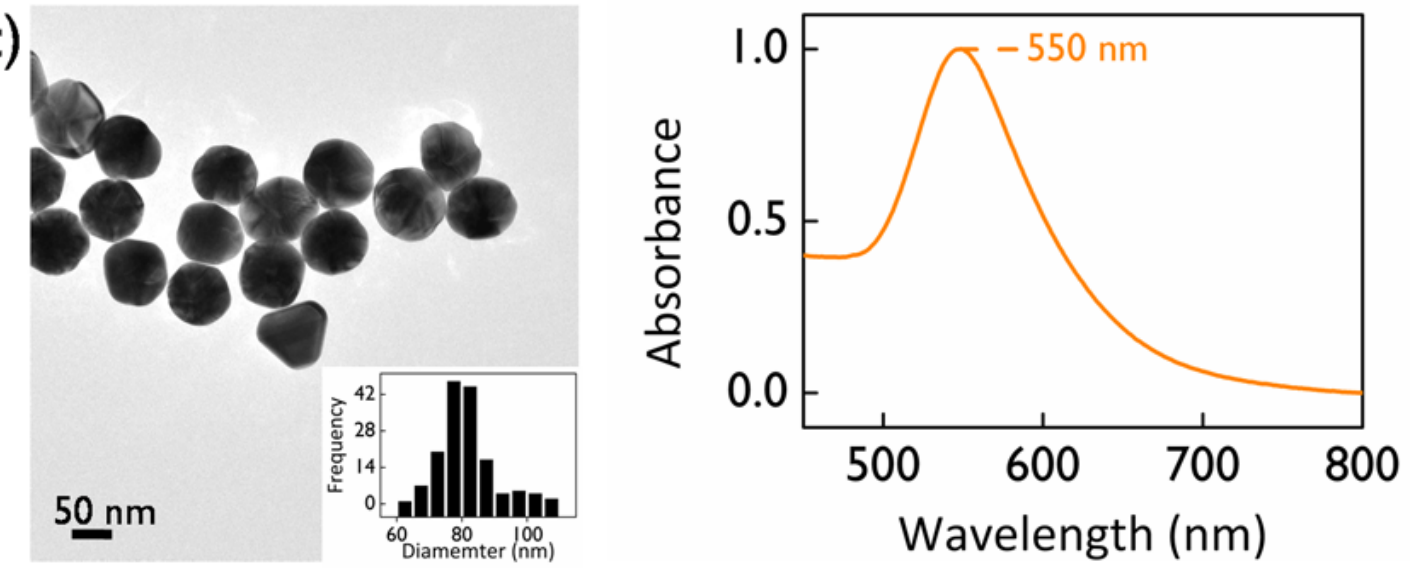

Figure S4. The Transmission Electron Microscopy images (Left panel) and UV-vis spectra of gold nanoparticles (Right panel). (a) gold nanoparticles with a diameter of $20 \mathrm{~nm}$; (b) gold nanoparticles with a diameter of $40 \mathrm{~nm}$; (c) gold nanoparticles with a diameter of $80 \mathrm{~nm}$. Inset: the corresponding statistical analysis of the size distribution of the AuNP. 
(a)

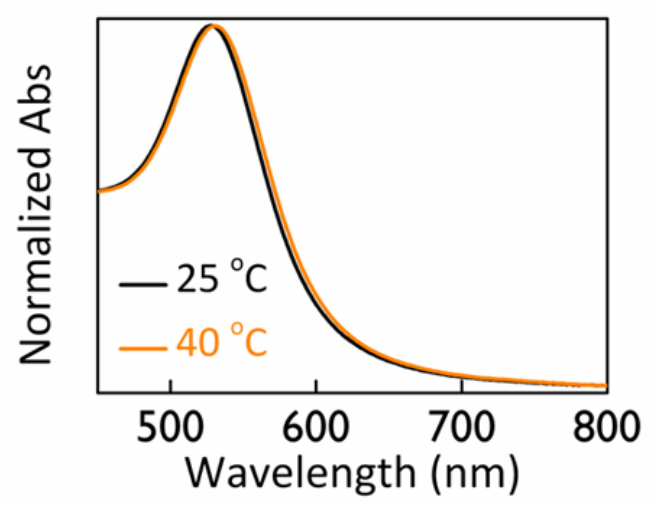

(b)

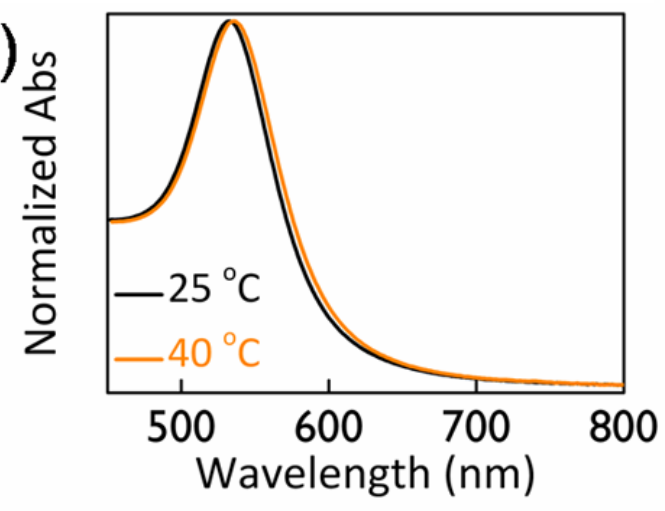

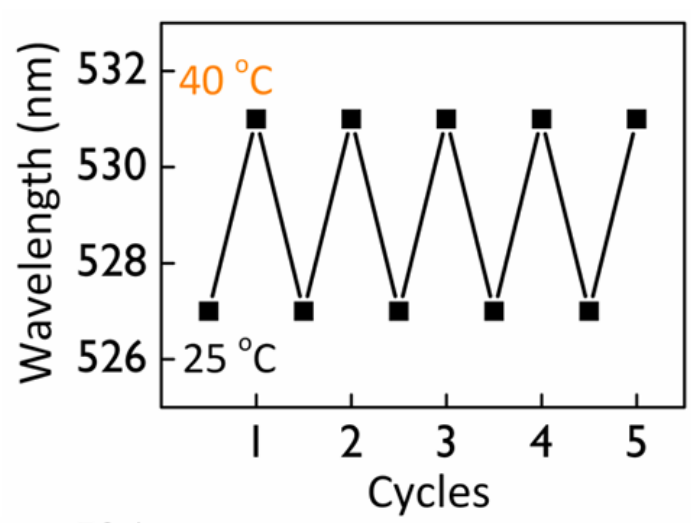

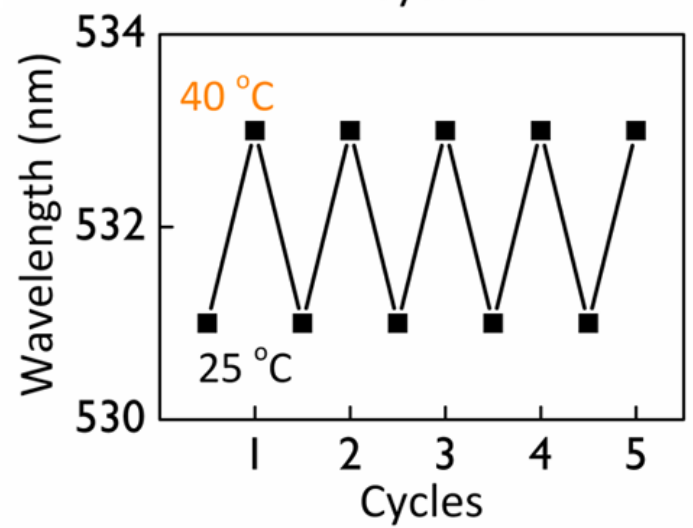

Figure S5. Representative UV-Vis Spectra characterizing the thermal behavior of the (a) pNIPAM@AuNP ${ }_{20}$, (b) pNIPAM@AuNP ${ }_{40}$. Left panel: Location of LSPR maxima monitored for AuNP $\left([\right.$ AuNP $\left.]=1.8 \times 10^{-8} \mathrm{mM}\right)$. Right Panel: the thermal cycling of the pNIPAM@AuNP. 
(a)

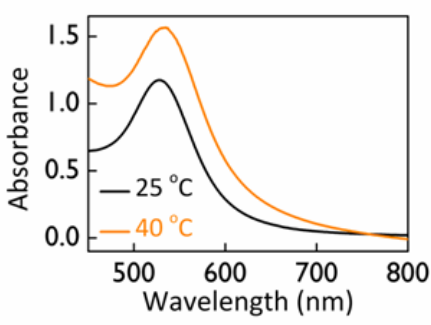

(b)

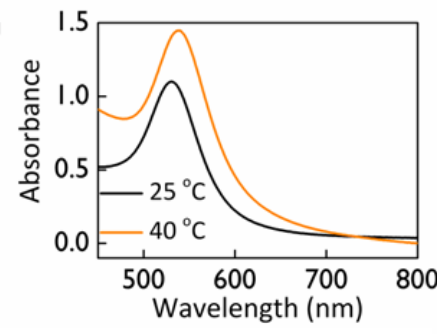

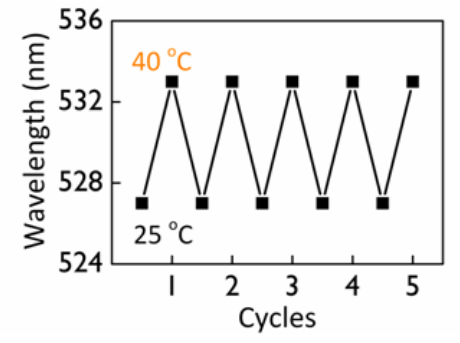

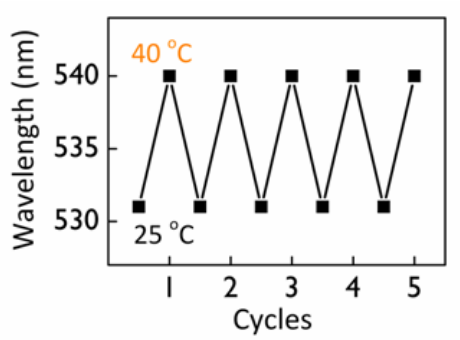

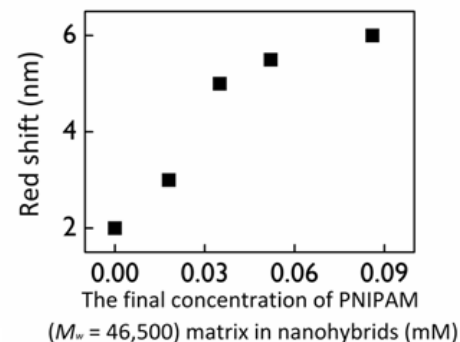

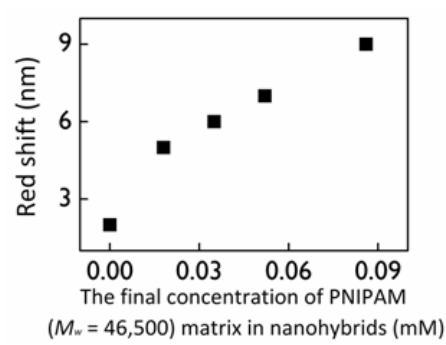

Figure S6. Representative UV-Vis characterizing the thermal behavior of the nanogels: (a) pNIPAM@AuNP 20 , (b) pNIPAM@AuNP 40 . Left Panel: Location of the LSPR maxima monitored for nanogels $\left([\mathrm{AuNP}]=1.8 \times 10^{-8} \mathrm{mM},[\mathrm{pNIPAM}]_{\text {matrix }}\right.$ ranging from 0 to $\left.0.086 \mathrm{mM}\right)$. Middle panel: the thermal cycling of the nanogels. Right panel: the red shift in the LSPR as a function of the final concentration of pNIPAM matrix in the nanogels. 


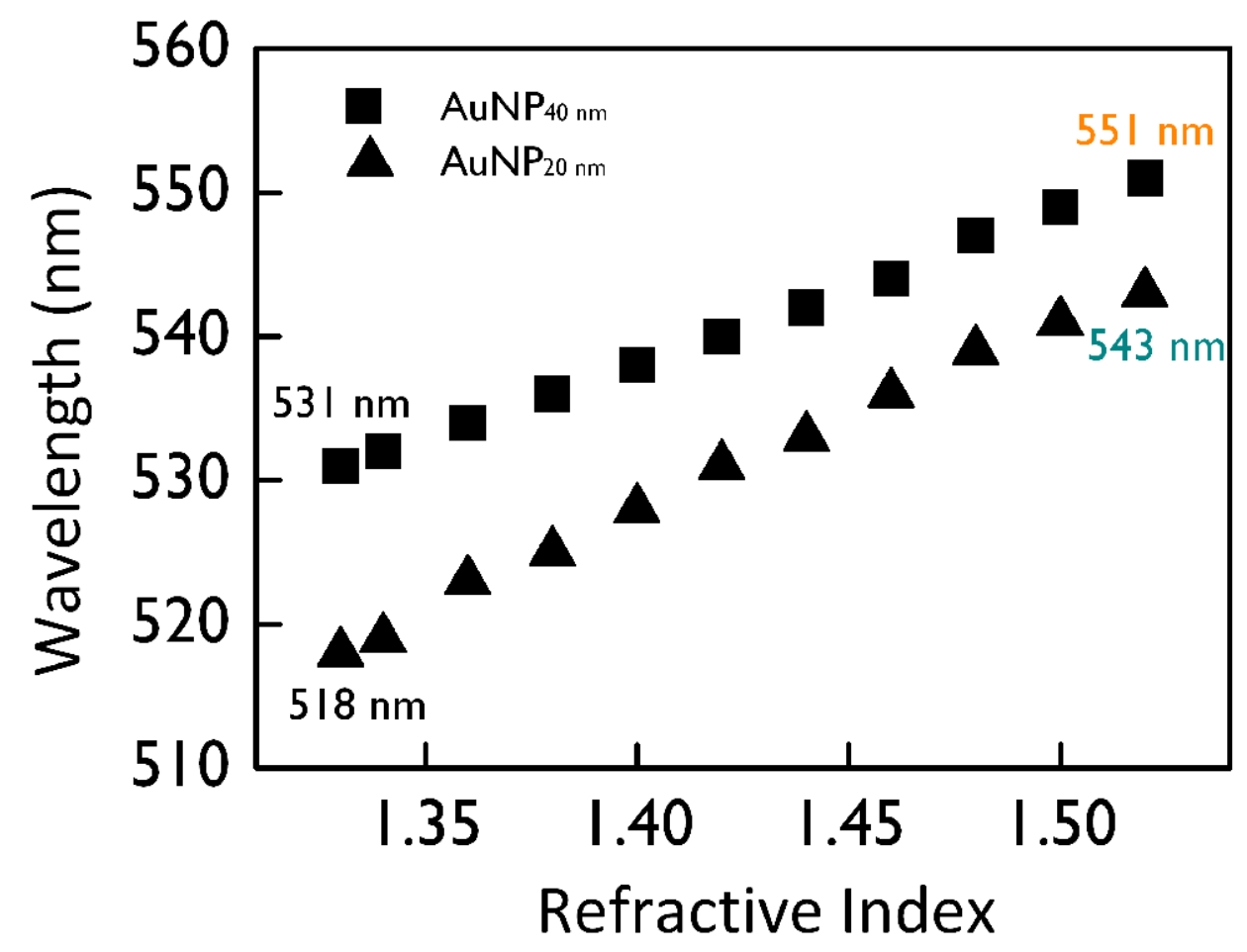

Figure S7. FDTD simulations of the shift in peak maxima of $\mathrm{AuNP}_{20}$, and $\mathrm{AuNP}_{40}$ in different refractive index media. 


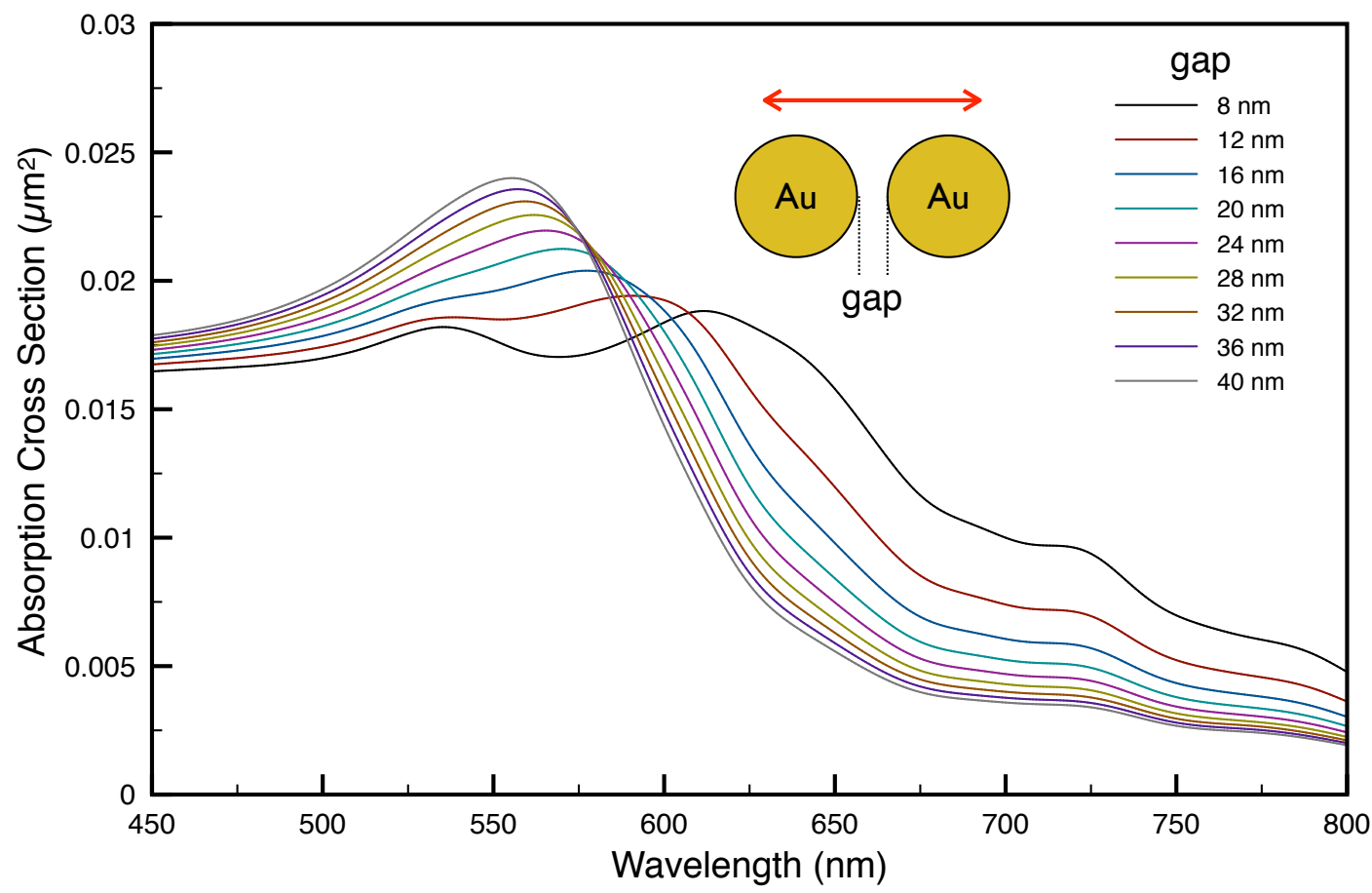

Figure S8. FDTD simulations of the absorbance spectra of two $\mathrm{AuNP}_{80}$ as a function of the spacing between the two particles with the polarization of light parallel to the dimer. 

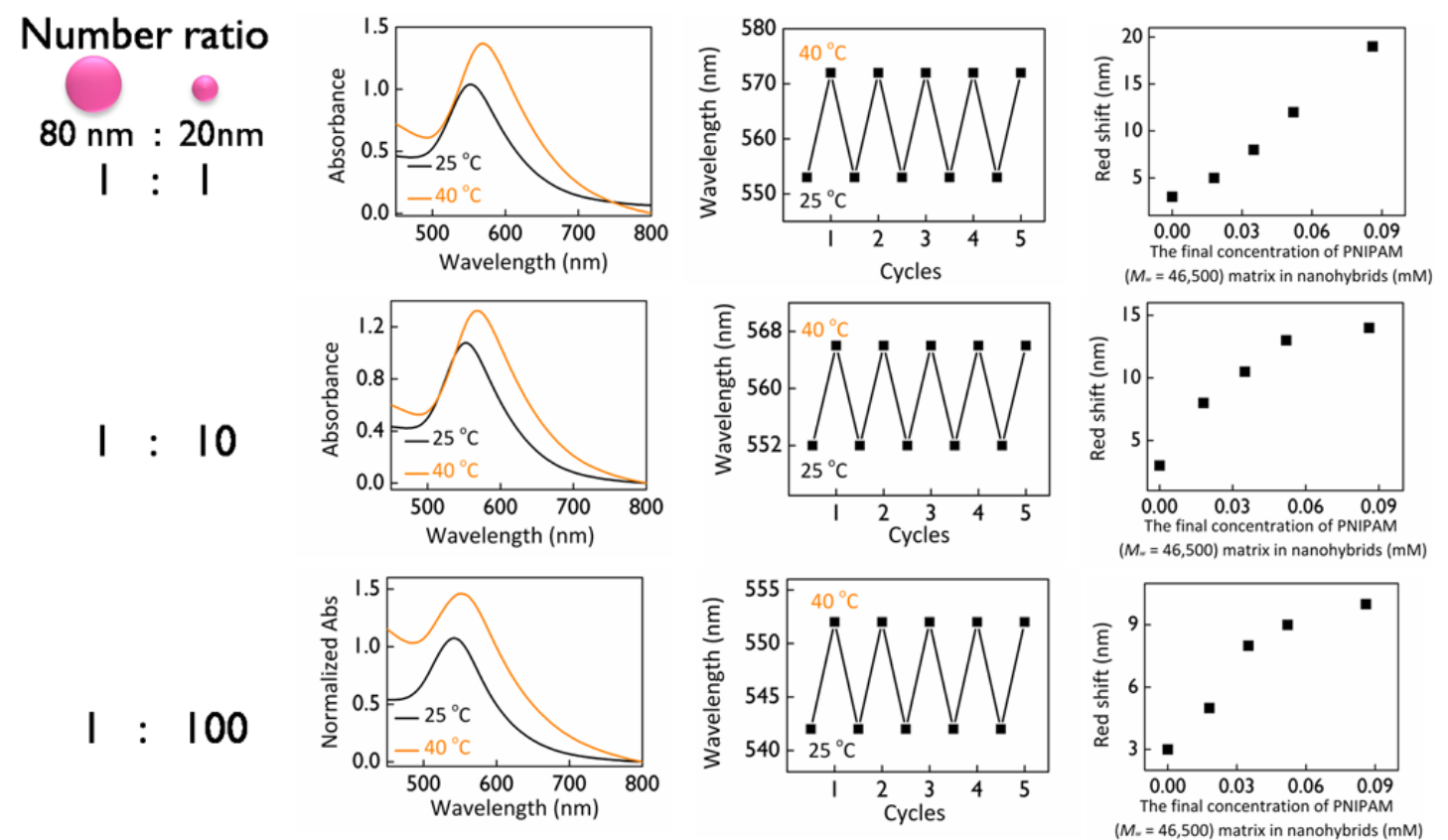

Figure S9. Representative UV-Vis characterizing the thermal behavior of the nanogels: pNIPAM@AuNP ${ }_{80}:$ pNIPAM@AuNP 20 at a number ratio of 1:1, 1:10 and 1:100. Middle Panel: Location of the LSPR maxima as a function of heating/cooling cycles $\left(\left[\mathrm{AuNP}_{80}\right]=1.8 \times 10^{-8} \mathrm{mM}\right.$, $\left[\mathrm{AuNP}_{20}\right]=1.16 \times 10^{-6} \mathrm{mM},[\mathrm{pNIPAM}]$ matrix ranging from 0 to $0.086 \mathrm{mM}$. Right panel: the red shift in the LSPR as a function of the final concentration of pNIPAM matrix in nanogels. 

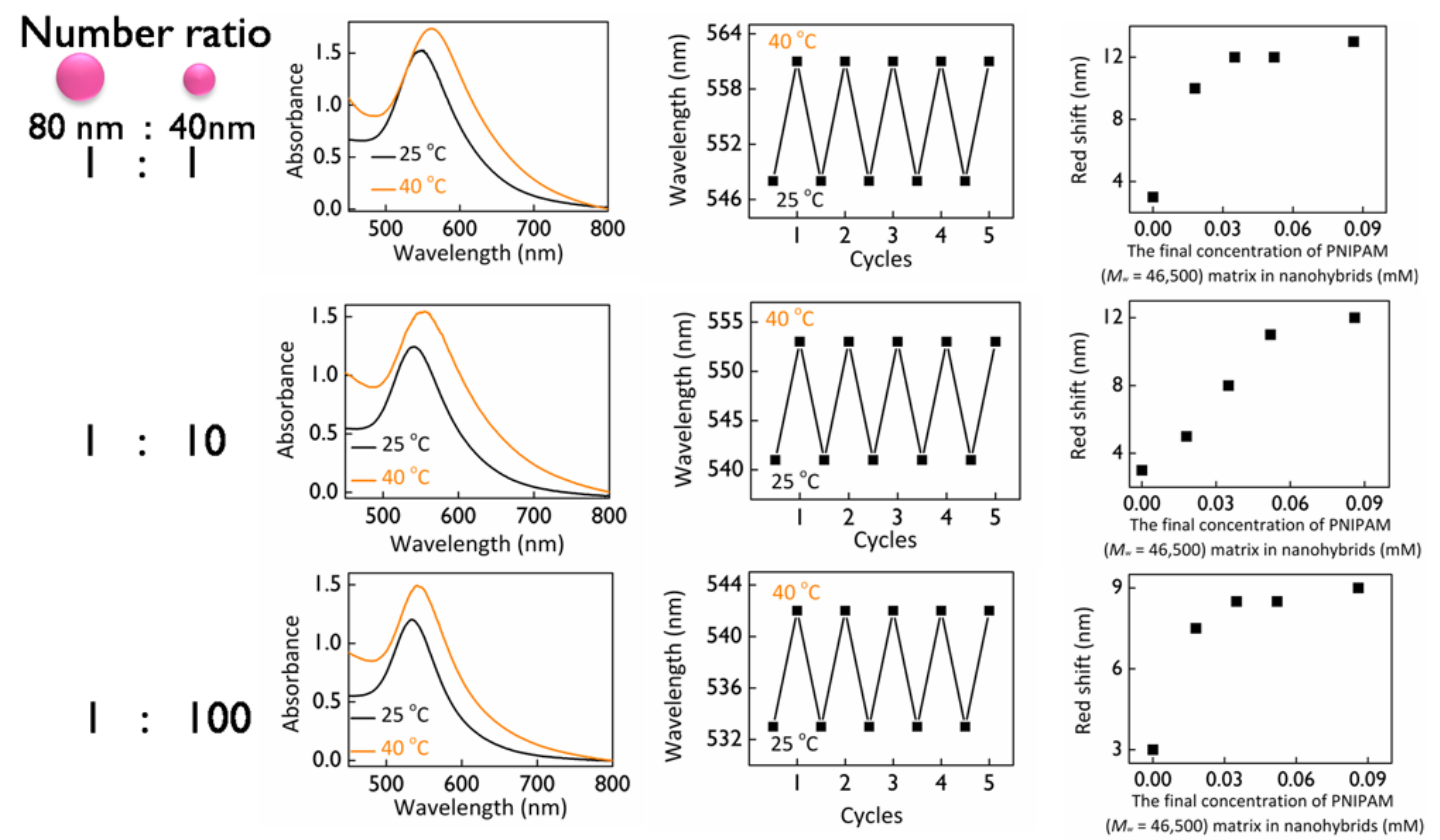

Figure S10. Representative UV-Vis characterizing the thermal behavior of the nanogels: pNIPAM@AuNP ${ }_{80}$ : pNIPAM@AuNP 40 at a number ratio of 1:1, 1:10 and 1:100. Middle Panel: Location of the LSPR maxima as a function of heating/cooling cycles $\left(\left[\mathrm{AuNP}_{80}\right]=1.8 \times 10^{-8} \mathrm{mM}\right.$, $\left[\mathrm{AuNP}_{40}\right]=1.5 \times 10^{-7} \mathrm{mM},[\mathrm{pNIPAM}]_{\text {matrix }}$ ranging from 0 to $0.086 \mathrm{mM}$. Right panel: The red shift was monitors at the increasing concentration of PNIPAM matrix. 


\section{Number ratio \\ $40 \mathrm{~nm}: 20 \mathrm{~nm}$ \\ I : I}
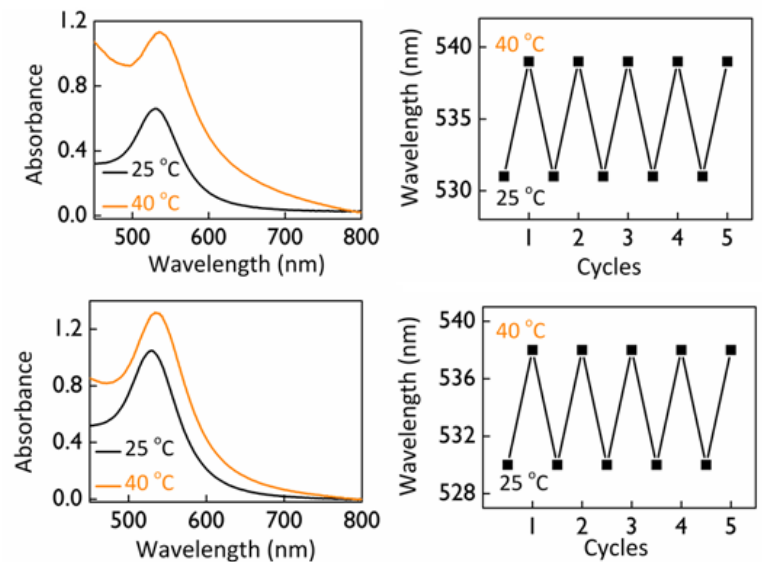

$1: 10$
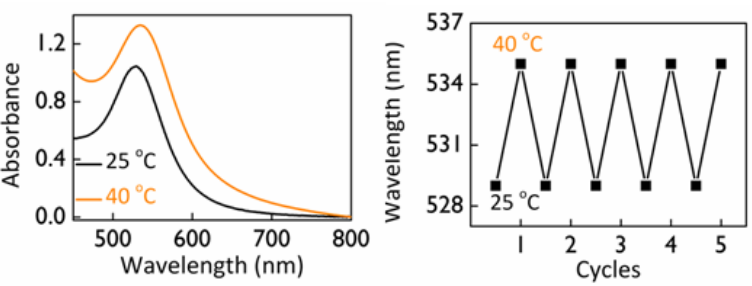
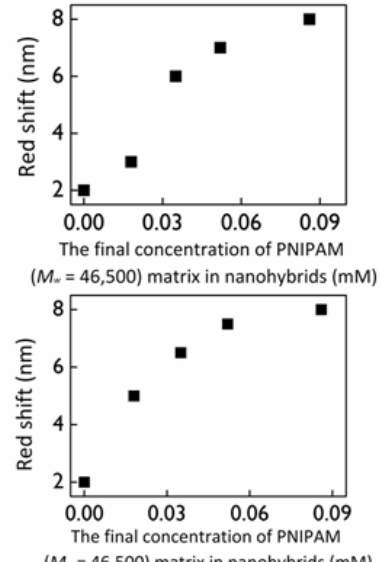

$\left(M_{*}=46,500\right)$ matrix in nanohybrids $(\mathrm{mM})$

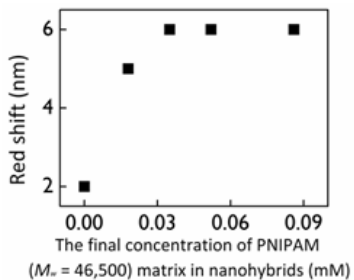

Figure S11. Representative UV-Vis characterizing the thermal behavior of the nanogels: pNIPAM@AuNP 40 : pNIPAM@AuNP 20 at a number ratio of 1:1, 1:10 and 1:100. Middle Panel: Location of the LSPR maxima as a function of heating/cooling cycles $\left(\left[\mathrm{AuNP}_{20}\right]=1.16 \times 10^{-6} \mathrm{mM}\right.$, $\left[\mathrm{AuNP}_{40}\right]=1.5 \times 10^{-7} \mathrm{mM}$, [pNIPAM $]_{\text {matrix }}$ ranging from 0 to $0.086 \mathrm{mM}$. Right panel: The red shift was monitors at the increasing concentration of pNIPAM matrix. 